\title{
Changes in Membrane Excitability and Potassium Currents in Sensitized Dorsal Horn Neurons of Mice Pups
}

\author{
Ivan Rivera-Arconada and Jose A. Lopez-Garcia \\ Department of Physiology, University of Alcala, Alcala de Henares, 28871 Madrid, Spain
}

Rationally, an increased intrinsic excitability of dorsal horn neurons could be a factor contributing to alter the gain of the nociceptive system during central sensitization, however direct evidence is scarce. Here we have examined this hypothesis using current and voltageclamp recordings from dorsal horn neurons in the spinal cord in vitro preparation obtained from mice pups of either sex. Cords were extracted from carrageenan-pretreated and control animals to allow for comparison. Dorsal horn neurons from treated animals showed significantly larger and faster synaptic responses. Synaptic changes started developing shortly after inflammation $(1 \mathrm{~h})$ and developed further after a longer-term inflammation $(20 \mathrm{~h})$. However, these neurons showed biphasic changes in membrane excitability with an increase shortly after inflammation and a decrease in the longer term. Concomitant changes were observed in transient $\left(I_{\mathrm{A}}\right)$ and sustained potassium currents $\left(I_{\mathrm{DR}}\right)$. Prolonged superfusion of naive spinal cords with NMDA led to a decreased neuronal excitability and to increased potassium currents.

Results suggest that excitability plays a role more complex than expected during the process of central sensitization of dorsal horn neurons and that modulation of potassium currents may contribute to shape the changing states of excitability. The decreased excitability observed after long-term inflammation is interpreted as a homeostatic correction to an abnormal state of synaptic activity.

\section{Introduction}

Damage to peripheral tissue causes strong activation of nociceptive primary afferents and a subsequent barrage of incoming information to the spinal cord. As a consequence of this increased input, the spinal cord can suffer plastic changes leading to an increased excitability of nociceptive circuits in a phenomenon that was termed central sensitization (Woolf, 1983, 2007). The nature of the changes that cause sensitization of spinal neurons is under discussion at present.

The incoming afferent barrage releases a variety of transmitters in the cord, and these may trigger modulation of synapses, membrane receptors, ion channels, and transcriptional processes (Ji and Woolf, 2001). There is substantial evidence indicating that modulation of NMDA receptors and synaptic plasticity may be fundamental factors involved in the generation of central sensitization (Liu and Sandkühler, 1995; Baba et al., 1999; Woolf and Salter, 2000). However, few studies have addressed the role that membrane excitability may play in central sensitization despite the fact that this is an obvious potential factor (Sandkühler et al., 2000).

Ion channel regulation in long-lasting pain states may include fast posttranscriptional events as well as slower processes leading to synthesis and degradation of channels (Woolf and Costigan, 1999). Baba et al. (1999) showed that basic membrane properties

Received Sept. 3, 2009; revised March 4, 2010; accepted March 10, 2010.

Research was supported by the Spanish Ministry of Science and Education (Grant No. SAF 2009-07876) and the Local Government of Madrid (Grant No. S-SAL 0305-2006).

Correspondence should be addressed to Jose A. Lopez-Garcia, Departamento Fisiología, Edificio de Medicina, Universidad de Alcala, Alcala de Henares, 28871 Madrid, Spain. E-mail: josea.lopez@uah.es.

DOI:10.1523/JNEUROSCI.4359-09.2010

Copyright $\odot 2010$ the authors $\quad 0270-6474 / 10 / 305376-08 \$ 15.00 / 0$ of dorsal horn neurons like membrane potential and resistance did not change during central sensitization. However, recent evidence indicates that $\mathrm{Kv} 4.2$ channels underlay A-type currents in mice dorsal horn neurons and that the genetic elimination of this channel prevents a normal hyperalgesic response to inflammatory stimuli (Hu et al., 2006). Transient and sustained potassium currents (A-type and delayed rectifier type currents) influence membrane excitability and are modulated by activity-dependent mechanisms (Desai et al., 1999; Yoshimura and de Groat, 1999; Stewart et al., 2003; Dang et al., 2004; Misonou et al., 2004; Takeda et al., 2006; van Welie et al., 2006; Xu et al., 2006; Wang et al., 2007; Lei et al., 2008; Shen et al., 2008).

Here we investigated the changes that a peripheral inflammation may cause in the intrinsic excitability of dorsal horn neurons and in potassium currents that are known to regulate neuronal excitability. We injected carrageenan in the hindpaws of mice pups to produce inflammation and hyperalgesia, a model shown to produce central sensitization in rat pups $20 \mathrm{~h}$ after inflammation (Hedo et al., 1999). Then we extracted the spinal cord of these animals to study the electrical behavior of lumbar dorsal horn neurons in their intact synaptic environment. We found that intrinsic excitability and the studied potassium currents undergo complex changes which may contribute to increase and to decrease the excitability of the system on a time-dependent basis.

\section{Materials and Methods}

Experiments were performed on C57BL6 mice pups (7- to 12-d-old) of either sex weighing between 4.3 and $9.7 \mathrm{~g}$. Experiments were designed following European Union and Spanish Government regulations, and the experimental protocols were approved by the local Ethics Committee. 
A

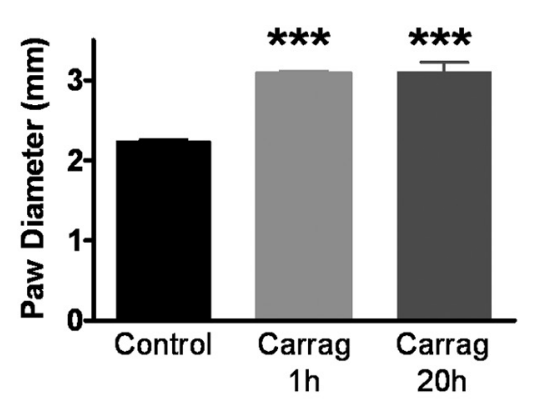

C

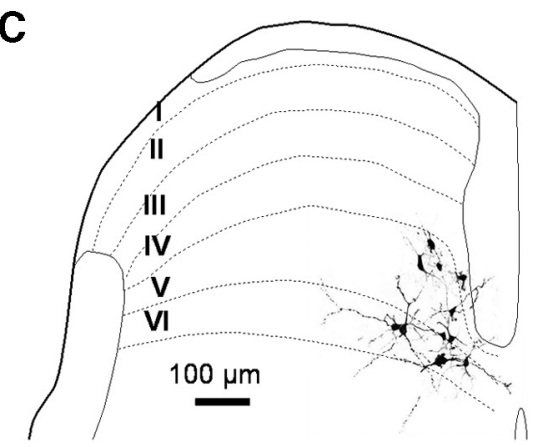

E

Control

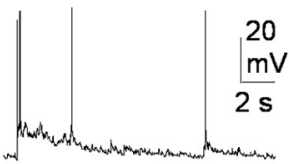

Carrageenan-20h
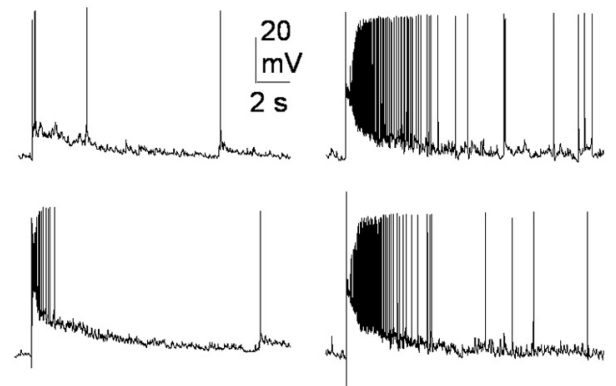

G

Control

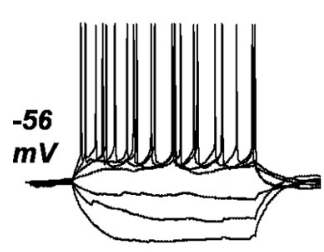

Carrageenan-20h
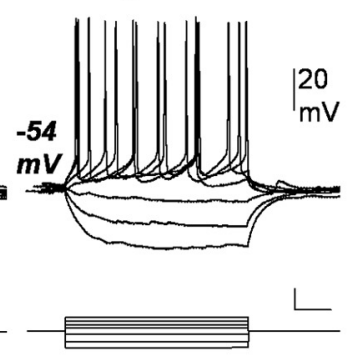

B

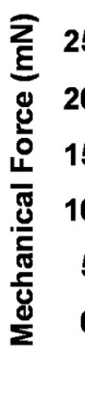

D
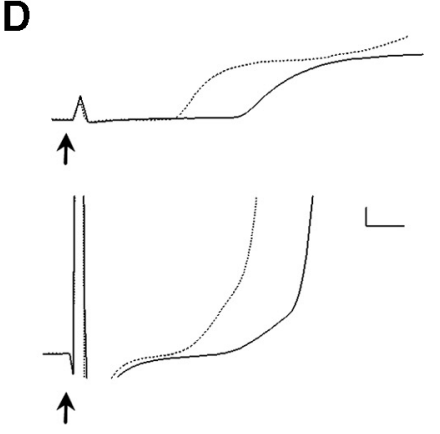

$\mathbf{F}$

Dorsal Root Stimulation

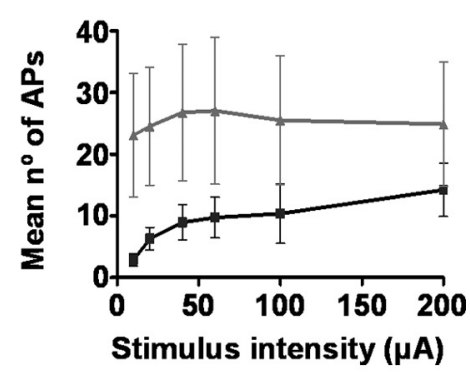

H Intracellular Pulses

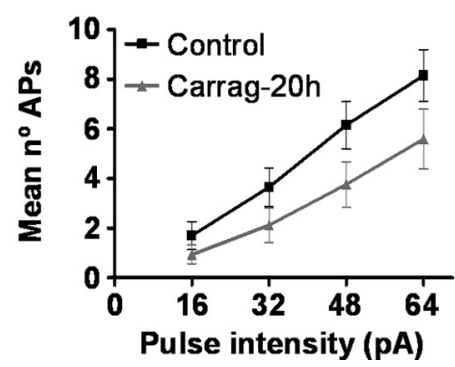

Figure 1. Behavioral assessment of inflammation and neuronal effects at $20 \mathrm{~h}$ posttreatment. $A$, Paw diameters measured in control and carrageenan-treated groups are shown. $\boldsymbol{B}$ shows withdrawal thresholds to punctuate mechanical stimuli applied with Von Fray filaments. Asterisks in $\boldsymbol{A}$ and $\boldsymbol{B}$ indicate significant differences (Mann-Whitney test; $p<0.001$ ). ( shows mayor morphological traits and laminar distribution of 11 neurons recovered after histological processing. Reconstructions are situated on a schematic drawing of a transverse section of the dorsal horn in which laminar boundaries are delineated and labeled with roman numbers. Voltage recordings shown in D correspond to the first milliseconds of neuronal responses to low intensity (200 $\mu$ s and threshold current; upper traces) and high intensity dorsal root stimulation (200 $\mu$ s and $200 \mu$ A, lower traces). Responses from control (continuous lines) are compared with those of injected with carrageenan 20 h earlier (doted lines). Resting membrane potential was $-54 \mathrm{mV}$ for all traces; cal. bars $4 \mathrm{mV}, 1 \mathrm{~ms}$. Voltage recordings in $\boldsymbol{E}$ show representative responses of dorsal horn neurons from control (left) and $20 \mathrm{~h}$ after carrageenan injection (right) to a low intensity stimulus ( $200 \mu$ s and $10 \mu \mathrm{A}$; top) and to high intensity stimulus ( $200 \mu \mathrm{s}$ and $200 \mu \mathrm{A}$; bottom) applied to the dorsal root (RMP $-58 \mathrm{mV}$ and $-57 \mathrm{mV}$, respectively). Graph in $\boldsymbol{F}$ shows quantitative data for neurons under control conditions (black squares, $n=19$ ) and after $20 \mathrm{~h}$ of inflammation (gray triangles, $n=16$ ). Difference between groups was significant ( $p<0.001 ;$ ANOVA). $\mathbf{G}$ shows responses to intracellular current injection obtain from neurons in control and treated conditions as indicated. Both figures show typical tonic firing patterns obtained in response to intracellular current pulses (unlabeled cal bars are $0.1 \mathrm{nA}$ and $0.1 \mathrm{~s}$ ). The graph in $\boldsymbol{H}$ shows pooled data on the action potentials elicited in response to intracellular current pulses in both groups (Control: black, $n=19$; carrageenan $20 \mathrm{~h}$ : gray, $n=$ 16). Difference between groups was significant ( $p<0.01$; ANOVA). Carrag, Carrageenan.

Induction and behavioral assessment of inflammation. Inflammation was induced by an intraplantar injection of carrageenan in both hindpaws $(30 \mathrm{mg} / \mathrm{ml}$ in saline; $20 \mu \mathrm{l}$ injection volume) 1 or $20 \mathrm{~h}$ before behavioral testing and spinal cord extraction. Paw caliber and mechanical withdrawal thresholds were measured just before spinal cord extraction using von Frey filaments as previously described (Hedo et al., 1999). Control animals did not receive any treatment before spinal cord extraction.

In vitro spinal cord preparation and dorsal root stimulation. Animals were anesthetized with urethane $(2 \mathrm{~g} / \mathrm{kg}$, i.p.) and their spinal cords extracted following a rostrocaudal laminectomy (Rivera-Arconada and Lopez-Garcia, 2005). Cords were hemisected and pinned down to a Sylgard-based recording chamber with the medial side upwards to facilitate electrode penetration. Spinal cords were maintained with oxygenated artificial CSF (ACSF) at room temperature $\left(22 \pm 1^{\circ} \mathrm{C}\right)$. The composition of the ACSF in mM was as follows: $\mathrm{NaCl}$ 127, $\mathrm{KCl} 1.9, \mathrm{KH}_{2} \mathrm{PO}_{4} 1.5, \mathrm{MgSO}_{4} 1.3, \mathrm{CaCl}_{2} 2$, $\mathrm{NaHCO}_{3} 22$, glucose 10, pH 7.4.

A lumbar dorsal root (L4-L5) was placed in a tight-fitting glass suction electrode and electrically stimulated to activate afferent fibers. Electrical stimuli consisted of a series of pulses of $200 \mu$ s duration and increasing intensities (10, $20,40,60,100$ and $200 \mu \mathrm{A}$ ) to recruit progressively the different primary afferent types within the root (Martinez-Gomez and LopezGarcia, 2005).

Current and voltage-clamp recordings. Electrodes were pulled from borosilicate glass tubing with internal filament using a horizontal puller (Sutter Instruments). Pipette resistances were within the range of 6-9 $\mathrm{M} \Omega$ when filled with the internal solution consisting of the following (in $\mathrm{mM}$ ): $\mathrm{KCl} 30$, EGTA 3, HEPES 40, $\mathrm{MgCl}_{2} 2$, potassium acetate $95, \mathrm{CaCl}_{2} 0.5, \mathrm{Na}_{2}-$ ATP 3, Na-GTP 0.3, pH 7.4. Electrodes were positioned under direct visual control on top of the lumbar dorsal horn gray matter and moved down by means of a micromanipulator while applying a positive pressure to the inside of the electrode. Signals were amplified with a MultiClamp 700A amplifier (Molecular Devices) and analyzed offline with Spike 2 and Signal software (CED, Cambridge Electronic Design). Current and voltage-clamp recordings were performed in independent samples of neurons.

Current-clamp recordings were digitized at $10 \mathrm{kHz}$. Input resistance was calculated using negative current pulses of low intensity $(<100$ $\mathrm{pA}$ ). Intracellular current pulses of $500 \mathrm{~ms}$ injected via the recording pipette were applied in increments of $16 \mathrm{pA}$ at $2 \mathrm{~s}$ intervals to study the intrinsic excitability and the firing patterns of neurons.

Voltage-clamp recordings were filtered at 4 $\mathrm{kHz}$, sampled at $10 \mathrm{kHz}$ and stored for off-line analysis. Recordings were performed in the presence of $0.5 \mu \mathrm{M}$ tetrodotoxin to block sodium currents and $100 \mu \mathrm{M}$ cadmium chloride to block calcium and calcium-activated potassium currents. Series resistances below $30 \mathrm{M} \Omega$ 
Table 1. Synaptic and electrophysiological properties of current clamp recorded dorsal horn neurons

\begin{tabular}{|c|c|c|c|c|}
\hline & Control & Carrageenan-treated $20 \mathrm{~h}$ & NMDA-treated & Carragenan-treated 1 \\
\hline \multicolumn{5}{|l|}{ Synaptic properties } \\
\hline Spontaneous firing $(n)$ & $9 / 19$ & $14 / 16^{*}$ & $4 / 9$ & $5 / 9$ \\
\hline \multicolumn{5}{|l|}{ A-fiber intensity } \\
\hline$n$ & 16 & 15 & 7 & 9 \\
\hline EPSP latency (ms) & $4.6 \pm 0.4$ & $3.3 \pm 0.2^{* *}$ & $3.9 \pm 0.7$ & $3.2 \pm 0.2^{*}$ \\
\hline Spike latency (ms) & $8.9 \pm 1.1$ & $5.2 \pm 0.5^{* *}$ & $6.2 \pm 1.2$ & $5.5 \pm 1.5^{*}$ \\
\hline Rise rate (mV/ms) & $5.8 \pm 1.0$ & $11.9 \pm 1.7^{* *}$ & $12.1 \pm 2.6^{*}$ & $14.6 \pm 2.4^{* *}$ \\
\hline \multicolumn{5}{|l|}{ C-fiber intensity } \\
\hline$n$ & 14 & 12 & 3 & 5 \\
\hline EPSP latency (ms) & $4.1 \pm 0.2$ & $3.3 \pm 0.2^{*}$ & $3.0 \pm 0.6$ & $2.9 \pm 0.2^{* *}$ \\
\hline Spike latency (ms) & $6.7 \pm 0.7$ & $4.7 \pm 0.4^{*}$ & $5.8 \pm 2.2$ & $3.8 \pm 0.3^{*}$ \\
\hline Rise rate (mV/ms) & $8.7 \pm 1.4$ & $17.4 \pm 2.7^{*}$ & $12.0 \pm 6.7$ & $17.1 \pm 2.7^{*}$ \\
\hline \multicolumn{5}{|c|}{ Electrophysiological properties } \\
\hline$n$ & 19 & 16 & 9 & 9 \\
\hline $\mathrm{RPM}(\mathrm{mV})$ & $-55.7 \pm 0.8$ & $-56.9 \pm 0.8$ & $-57.3 \pm 1.9$ & $-58.4 \pm 1.2$ \\
\hline$R_{\text {in }}(\mathrm{M} \Omega)$ & $355 \pm 20$ & $327 \pm 30$ & $348 \pm 35$ & $426 \pm 33$ \\
\hline $\mathrm{AP}-\mathrm{A}(\mathrm{mV})$ & $65 \pm 2$ & $71 \pm 3$ & $72 \pm 3$ & $63 \pm 3$ \\
\hline AP-T $(m V)$ & $-39.7 \pm 0.7$ & $-39.7 \pm 0.6$ & $-39.6 \pm 1.0$ & $-44.7 \pm 1.1^{* * *}$ \\
\hline Tonic firing $(\mathrm{n})$ & 18 & 13 & 8 & 8 \\
\hline Phasic firing (n) & 1 & 3 & 1 & 1 \\
\hline
\end{tabular}

Quantifications of synaptic properties were made on responses to A- and C-fiber intensity stimuli ( $200 \mu$ s and $20 \mu \mathrm{A}$, and $200 \mu$ s and $200 \mu \mathrm{A}$, respectively). Values are means \pm SEM. Asterisks mark significant differences with control ( ${ }^{*} p<0.05$ ${ }^{* *} p<0.01 ;{ }^{* * *} p<0.001$, Mann-Whitney test or Fisher exact test). $n$, Number of observations; RMP, resting membrane potential; $R_{\text {in }}$, input resistance; AP-A, action potential amplitude; AP-T, action potential threshold.

were routinely obtained and were used to correct voltage values offline. Electrode capacitance was reduced by coating the pipette tip with Sylgard. Membrane time constant was calculated fitting an average of at least 10 voltage steps from $-60 \mathrm{mV}$ to $-70 \mathrm{mV}$ to a double exponential function. Cell capacitance was calculated dividing the time constant by the input resistance.

Transient potassium currents were isolated by a two-step voltage protocol as previously described by others (Hu et al., 2003). Briefly, from a holding voltage of $-80 \mathrm{mV}$ a series of $500 \mathrm{~ms}$ depolarizing step commands were applied in $+10 \mathrm{mV}$ increments to a maximum of $+50 \mathrm{mV}$. The corresponding paired pulse consisted of a $150 \mathrm{~ms}$ prepulse command to $-10 \mathrm{mV}$ followed by a $500 \mathrm{~ms}$ step command to the corresponding final voltage. Voltage stimuli were applied at $5 \mathrm{~s}$ intervals. Current intensities for the transient component were analyzed in currents resulting from the digital subtraction of each pair of pulses. Sustained currents were measured within the last $50 \mathrm{~ms}$ of the voltage step after off-line leak subtraction.

Determination of voltage-dependent inactivation of transient currents was performed using incremental conditioning command prepulses of $150 \mathrm{~ms}$ duration from -100 to $+30 \mathrm{mV}$, followed by a voltage step command to $+40 \mathrm{mV}$ for $500 \mathrm{~ms}$ (Hu et al., 2003). Recovery from inactivation of transient currents was studied using $500 \mathrm{~ms}$ paired step commands to $+40 \mathrm{mV}$ with an incremental delay between them (starting at $3 \mathrm{~ms}$ ).

Intracellular labeling and tissue processing. On completion of experimental protocols biocytin $(0.5 \mathrm{mg} / \mathrm{ml})$ was electrophoresed into the neuron using continuous hyperpolarizing current $(-0.6$ to $-0.8 \mathrm{nA}$ for $15-20 \mathrm{~min})$. A $2-3 \mathrm{~mm}$ portion of the hemisected cord containing the filled neuron was sectioned and placed in cold fixative (4\% paraformaldehyde) overnight, followed by $24 \mathrm{~h}$ in a $30 \%$ sucrose-containing phosphate buffer saline. Tissue processing was performed as previously reported (Rivera-Arconada and Lopez-Garcia, 2005). Body size, general morphological traits, and position of the recovered neurons were recorded.

Drugs and chemicals. Carrageenan lambda, $N$-methyl-D-aspartic acid (NMDA), cadmium chloride $\left(\mathrm{CdCl}_{2}\right)$, tetraethylammonium (TEA), 4-aminopyridine (4-AP), biocytin, and the components for the ACSF and the intracellular solutions were purchased from Sigma-Aldrich. Tetrodotoxin (TTX) was purchased from Tocris Bioscience.

The ACSF was freshly prepared for each experiment. The intracellular solution was stored in aliquots at $-20^{\circ} \mathrm{C}$. For experiments, an aliquot was defrosted, and ATP and GTP were freshly added just before the beginning of the recording session.
Drugs were dissolved in ultrapure water as concentrated stocks and stored in aliquots at $-20^{\circ} \mathrm{C}$, except for TEA which was diluted directly in ACSF. All compounds were diluted in ACSF to the final concentration just before use.

Data analysis. All statistical analysis and curve fittings were performed using GraphPad Prism 4.0. Responses to dorsal root stimulation and intracellular pulses were quantified in terms of number of action potentials against intensity of dorsal root stimulus or intracellular pulse, respectively, and the resulting data were analyzed using two-way ANOVA.

Activation curves were constructed converting current values at each corrected test potential to conductances using the following equation with a calculated $E_{\mathrm{K}}$ of $-94 \mathrm{mV}: g=I /\left(V-E_{\mathrm{K}}\right)$. Normalized conductances were fitted using a Boltzmann function: $g / g_{\max }=$ Bottom + (Top - Bottom $) /$ $\left(1+\exp \left(\left(V_{50}-V\right) /\right.\right.$ Slope $\left.)\right)$, where $g_{\max }$ was the maximum conductance measured at maximum voltage attained, $V$ the corrected test potential and $V_{50}$ the potential for half-activation.

Inactivation curves of isolated transient currents were constructed using normalized amplitudes fitted to the Boltzmann equation:

$$
I / I_{\max }=\text { Bottom }+(\text { Top }- \text { Bottom }) /\left(1+\exp \left(\left(V_{50}-V\right) / \text { Slope }\right)\right),
$$

where $I_{\max }$ was the maximum current obtained from the more hyperpolarized prepulse potential, $V$ the corrected test potential, and $V_{50}$ the potential for half-inactivation.

Direct data on current densities for transient currents were fitted to the Boltzmann model as well. Current density for sustained currents did not fit to the Boltzmann model and voltage values $\geq-18 \mathrm{mV}$ were fitted to straight lines. Comparisons between groups were made using mean $V_{50}$ and/or slope values using the Mann-Whitney test. Reactivation curves for transient currents were fitted to a hyperbolic function.

\section{Results}

\section{Central effects of a long-term peripheral inflammation}

Twenty hours after carrageenan injections, animals showed peripheral inflammation and mechanical allodynia (Fig. $1 A, B$ ). Currentclamp recordings using control and treated animals were performed on a sample of electrophysiologically similar neurons situated in laminae IV-VI of the dorsal horn (Table 1, Fig. 1C).

Neurons recorded from carrageenan-treated mice showed clear signs of sensitization which reflected in significantly shorter latencies and faster depolarization rates in responses to dorsal root stimulation as well as in the presence of suprathreshold spontaneous activity which was more frequent than in control 


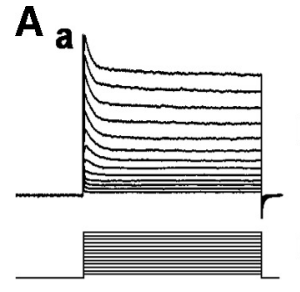

C

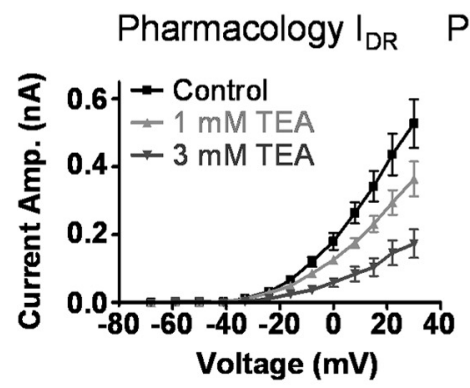

D

Pharmacology $I_{A}$

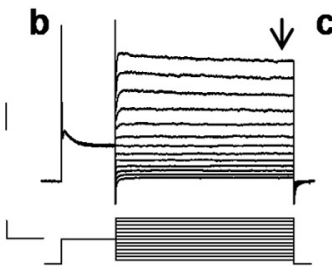

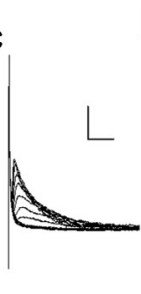

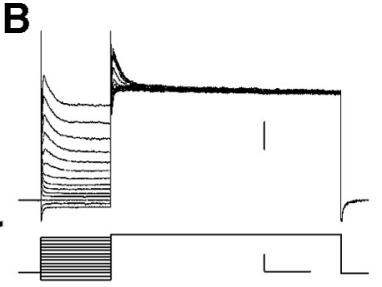

E
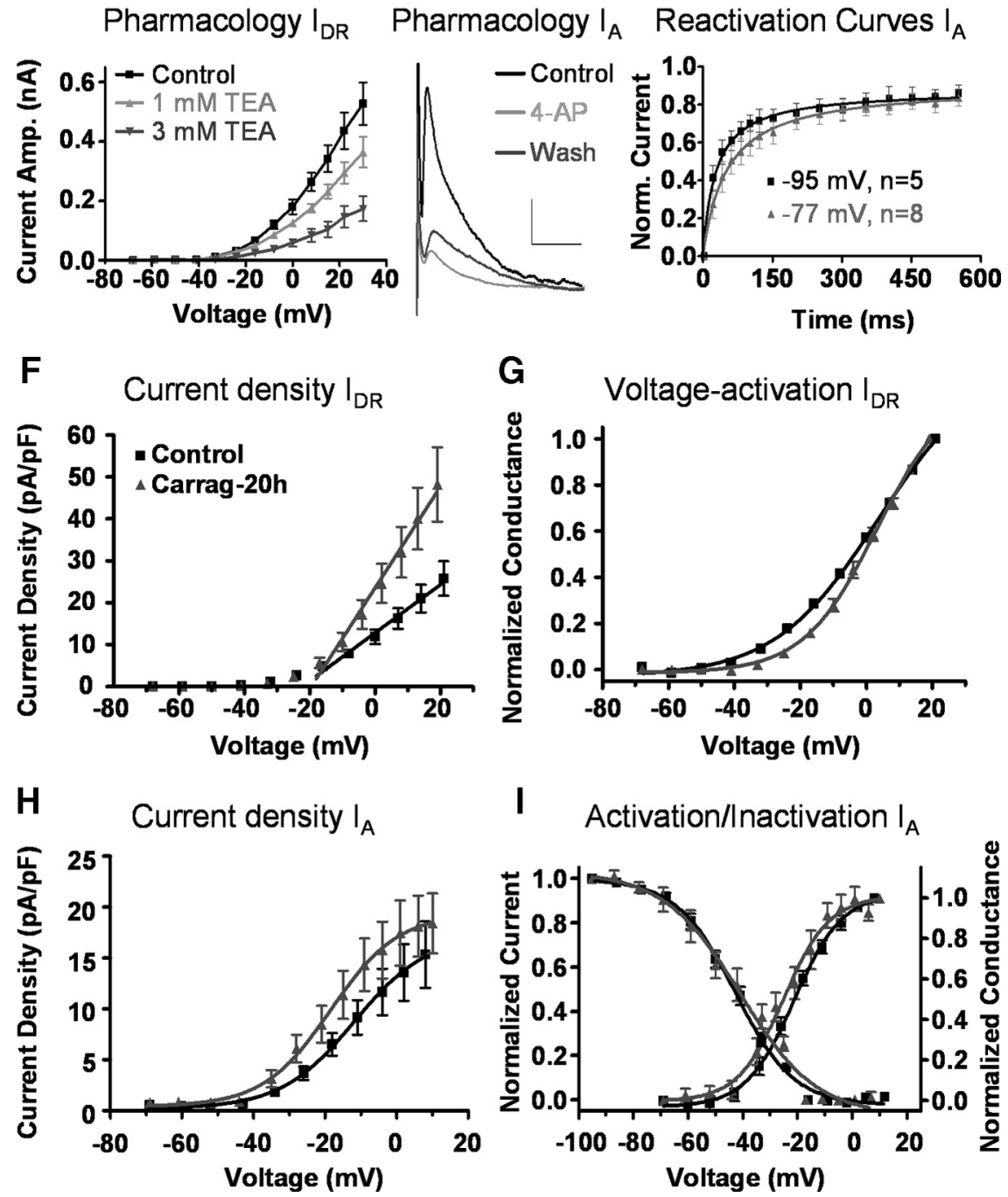

Figure 2. Effects of a long-term inflammation $(20 \mathrm{~h})$ on potassium currents. A shows original recordings of outward potassium currents in response to a series of depolarizing voltage steps. Both transient and sustained currents were activated by voltage steps from a holding of $-80 \mathrm{mV}(\mathbf{A a})$. With a prepulse command to $-10 \mathrm{mV}$ for $150 \mathrm{~ms}$, transient currents inactivate and in the subsequent step command to each test potential only sustained currents remain $(\boldsymbol{A} \boldsymbol{b})$. Transient currents were isolated by subtraction of both traces at each test voltage $(\boldsymbol{A c})$. Sustained currents were measured at the end of the voltage pulse (black arrow). Original recordings in $\boldsymbol{B}$ illustrate voltage-dependent inactivation of transient currents as obtained from a representative neuron. Upper traces show the current response to the application of a series of voltage step commands to potentials between -100 and $+30 \mathrm{mV}$ followed by a test potential command to $+40 \mathrm{mV}$ (lower traces). Sustained currents were sensitive to 1 and $3 \mathrm{~mm}$ TEA as shown in $C(n=3$; overall ANOVA $p<0.001)$. The transient current was identified as the $I_{A}$ since brief applications of $5 \mathrm{~mm}$ 4-AP reversibly blocked transient currents to $41.2 \pm 2.8 \%$ of control as shown in $\boldsymbol{D}(n=5)$, and it displays a rapid recovery from inactivation typical of A-type currents, a property enhanced at more negative holding potentials as shown in $\boldsymbol{E}$ (corrected holding potentials as indicated in figure). Calibration bars for $\boldsymbol{A} \boldsymbol{a}, \boldsymbol{A b}$, and $\boldsymbol{B}$ correspond to $0.5 \mathrm{nA}, 50 \mathrm{mV}$, and $100 \mathrm{~ms}$; for $\boldsymbol{A c}, 0.5 \mathrm{nA}, 20 \mathrm{~ms}$; for $D, 0.2 \mathrm{nA}, 20 \mathrm{~ms}$. Current density for $I_{\mathrm{DR}}$ was greater in neurons from carrageenan-treated mice $(n=8)$ than in control neurons $(n=21)$ as shown in $\boldsymbol{F}$ (slope of straight line fit, $p<0.05)$. The color code applies to all graphs. Graph in $\boldsymbol{G}$ shows the activation curve for $I_{D R}$ in the same sample of neurons shown in $\boldsymbol{F}$. After inflammation, the curve was displaced to more depolarized voltages, and the slopes of the Boltzmann sigmoid fits were significantly different $(p<0.001)$. Density of transient currents $(\boldsymbol{H})$ and voltage-dependent activation and inactivation curves $(\boldsymbol{I})$ were not affected by treatment. Carrag, Carrageenan. neurons (Fig. 1D, Table 1). Responses to graded dorsal root stimulation were significantly greater after treatment (Fig. $1 E, F)$. In control neurons, increasing the stimulus intensity applied to the dorsal root produced a clear increase in the number of elicited action potentials whereas neurons from carrageenan-treated animals showed responses with large numbers of action potentials which did not change with stimulus intensity.

To assess a possible contribution of intrinsic properties of neurons to the sensitization observed, we compared some basic membrane properties of neurons in control and carrageenan-treated animals (Table 1). No differences were found in resting membrane potential, input resistance and action potential threshold and amplitude.

Depolarizing pulses revealed the main patterns of firing adaptation previously described (Lopez-Garcia and King, 1994). In both control and treated animals, the large majority of neurons displayed tonic firing, but phasic and single spike neurons were also present in both samples. The frequency distribution of tonic versus phasic neuronal firing patterns did not change after inflammation (Table 1).

We compared the number of action potentials elicited by $500 \mathrm{~ms}$ pulses of increasing current intensity and, contrary to our expectations, found that neurons from carrageenan-treated animals produced a significantly smaller number of spikes than those from control mice (Fig. 1G,H).

This latter observation suggests that a long-lasting peripheral inflammation caused by carrageenan injection, induces changes in the expression or function of ionic conductances that decrease the excitability of spinal neurons. Since potassium currents are known to modulate neuronal excitability, we identified transient (A type or $I_{\mathrm{A}}$ ) and sustained outward potassium currents (delayed rectifier type or $I_{\mathrm{DR}}$ ) present in a new sample of neurons following previous procedures and criteria (Fig. 2A-E) (Rudy, 1988; Storm, 1990; Jerng et al., 2004). The basic electrophysiological properties of neurons in this sample are shown in Table 2.

The $I_{\mathrm{DR}}$ was significantly modified by the treatment. After a long-lasting peripheral inflammation, current density was larger and the voltage dependency for activation had a lower slope value compared with control but no changes were observed in $V_{50}$ (Fig. $2 F, G$, Table 2). In contrast, the main characteristics of transient currents were not modified by inflammation (Fig. 2H,I, Table 2). 
Table 2. Characterization of sustained $\left(I_{D R}\right)$ and transient $\left(I_{A}\right)$ currents under different experimental conditions

\begin{tabular}{|c|c|c|c|c|}
\hline & Control & Carrageenan-treated $20 \mathrm{~h}$ & NMDA-treated & Carrageenan-treated $1 \mathrm{~h}$ \\
\hline \multicolumn{5}{|l|}{ Basic electrophysiological properties } \\
\hline$n$ & 21 & 8 & 14 & 11 \\
\hline $\mathrm{RMP}(\mathrm{mV})$ & $-55.7 \pm 1.5$ & $-54.2 \pm 2.2$ & $-54.3 \pm 1.3$ & $-55.4 \pm 2.2$ \\
\hline$R_{\text {in }}(\mathrm{M} \Omega)$ & $445 \pm 42$ & $486 \pm 38$ & $360 \pm 34$ & $439 \pm 61$ \\
\hline Capacitance (pF) & $19.5 \pm 1.8$ & $16.5 \pm 2.0$ & $17.4 \pm 2.5$ & $15.6 \pm 2.6$ \\
\hline \multicolumn{5}{|l|}{ Sustained currents } \\
\hline$n$ & 21 & 8 & 14 & 11 \\
\hline Max. current density (pA/pF) & $25.7 \pm 4.1$ & $48.2 \pm 8.8^{*}$ & $78.0 \pm 13.9^{* *}$ & $55.2 \pm 18.6$ \\
\hline Max. voltage attained (mV) & 21 & 19 & 21 & 23 \\
\hline Slope activation & $0.6 \pm 0.1$ & $1.2 \pm 0.2^{*}$ & $1.7 \pm 0.3^{* *}$ & $1.1 \pm 0.4$ \\
\hline$V_{50}(\mathrm{mV})$ & $2.1 \pm 1.1$ & $2.6 \pm 1.4$ & $0.4 \pm 1.6$ & $-9.2 \pm 1.2^{* * *}$ \\
\hline$K$ & $15.1 \pm 0.5$ & $10.7 \pm 0.6^{* * *}$ & $13.5 \pm 0.6$ & $12.1 \pm 0.4^{* * *}$ \\
\hline \multicolumn{5}{|l|}{ Transient currents } \\
\hline$n$ & 18 & 8 & 11 & 7 \\
\hline Max. current density (pA/pF) & $15.3 \pm 3.3$ & $18.4 \pm 2.9$ & $39.0 \pm 13.1^{*}$ & $18.7 \pm 9.3$ \\
\hline Max. voltage attained (mV) & 8 & 10 & 10 & 8 \\
\hline$V_{50}(\mathrm{mV})$ & $-10.7 \pm 2.9$ & $-15.6 \pm 2.7$ & $-14.4 \pm 1.1$ & $-8.1 \pm 7.3$ \\
\hline K & $11.9 \pm 1.8$ & $10.8 \pm 2.0$ & $8.3 \pm 1.2^{*}$ & $12.0 \pm 1.7$ \\
\hline Time constant (ms) & $15.9 \pm 1.5$ & $15.7 \pm 2.1$ & $15.5 \pm 2.5$ & $27.2 \pm 9.3$ \\
\hline \multicolumn{5}{|l|}{ Activation } \\
\hline$V_{50}(\mathrm{mV})$ & $-22.1 \pm 1.8$ & $-24.2 \pm 2.7$ & $-20.4 \pm 1.2$ & $-22.0 \pm 3.6$ \\
\hline K & $9.3 \pm 0.9$ & $8.7 \pm 1.5$ & $7.1 \pm 1.1$ & $8.5 \pm 0.7$ \\
\hline \multicolumn{5}{|l|}{ Inactivation } \\
\hline$V_{50}(\mathrm{mV})$ & $-43.9 \pm 1.7$ & $-39.4 \pm 2.8$ & $-47.0 \pm 2.0$ & $-54.3 \pm 4.9$ \\
\hline$K$ & $-10.5 \pm 1.0$ & $-12.6 \pm 1.6$ & $-9.7 \pm 1.2$ & $-15.5 \pm 1.9^{*}$ \\
\hline
\end{tabular}

Values are means \pm SEM. Values for maximum (Max.) current density were obtained at the maximum voltage attained as indicated. Density of sustained currents for voltages over $-18 \mathrm{mV}$ were fitted to a straight line and slope values are given. For data fitted to the Boltzmann sigmoidal function, $V_{50}$, voltage of half-maximal activation or inactivation, and $K$, slope factor. The inactivation time constant was obtained fitting isolated transient currents obtained at $\approx+10 \mathrm{mV}$ to a single exponential function. Asterisks stand for statistical significance using Mann-Whitney test. Levels of confidence are as follows: ${ }^{*} p<0.05,{ }^{* *} p<0.01,{ }^{* * *} p<0.001 . n$, Number of neurons; RMP, resting membrane potential; $R_{\text {in },}$, input resistance.

These experiments show that potassium channels in dorsal horn neurons are susceptible to be modulated by processes triggered by peripheral inflammation. Under the present conditions the $I_{\mathrm{DR}}$ but not the $I_{\mathrm{A}}$ was significantly increased. In the light of data indicating that neuronal excitability can suffer homeostatic changes depending upon synaptic activity in various systems and brain areas (Desai et al., 1999; Misonou et al., 2004), we designed a second experiment to test whether this concept applied to the $I_{\mathrm{DR}}$ in dorsal horn neurons.

\section{Modulation of outward potassium currents by NMDA receptor activation}

NMDA receptors play a pivotal role in transmission along nociceptive pathways, and their activation has been shown to modulate potassium currents in other systems (Lei et al., 2008; Mulholland et al., 2008; Shen et al., 2008). Here we superfused the cord with NMDA (20 min) at a concentration sufficient to produce sustained firing $(10 \mu \mathrm{M})$ and then recorded dorsal horn neurons in a time window between 1 and $4 \mathrm{~h}$ after NMDA perfusion. Basic electrophysiological properties of these neurons were similar to those of controls (Tables 1,2).

Current-clamp recordings obtained from these neurons showed potentiated responses to dorsal root stimulation and a smaller number of action potentials to intracellular current pulses than neurons from control animals (Fig. $3 A, B$ ).

Voltage-clamp recordings showed a large and significant increase in current density for $I_{\mathrm{DR}}$ with no change in voltage dependency of activation (Fig. 3C,D). Similarly, NMDA perfusion produced a large and significant increase in current density for the $I_{\mathrm{A}}$ and no change in activation or inactivation kinetics (Fig. $3 E, F$, Table 2).

This experiment shows that a sustained activation of NMDA receptors can cause changes in neuronal excitability involving modulation of potassium currents in the dorsal horn. Results support the hypothesis that the $I_{\mathrm{DR}}$ may behave as a homeostatic correction for excessive synaptic input. However NMDA perfusion caused changes in $I_{\mathrm{A}}$ which did not reproduce the effects of a long-term inflammation.

If the increase in $I_{\mathrm{DR}}$ was a homeostatic correction, predictably the increase would be associated to the development of synaptic changes. In addition, previous studies suggest that inflammation may cause changes in $I_{\mathrm{A}}$ ( $\mathrm{Hu}$ et al., 2006). On these bases, we designed a third experiment to look at the short-term effects of inflammation on dorsal horn neurons.

\section{Central effects of a short-term peripheral inflammation}

To study short-term effects of inflammation, mice were treated with carrageenan $1 \mathrm{~h}$ before cord isolation and recordings were performed in the subsequent $4 \mathrm{~h}$. These animals showed peripheral inflammation and mechanical allodynia at the time of cord extraction (Fig. $1 A, B$ ).

Current-clamp recordings from dorsal horn neurones after short-term inflammation showed changes in their responses to dorsal root stimulation similar to those described for a longlasting inflammation including a reduction in latency and an increase in the number of elicited action potentials (Table 1, Fig. $4 A$ ). However the proportion of neurons showing spontaneous suprathreshold activity was similar to that of the control group (Table 1). Basic electrophysiological properties were similar to control neurons except for action potential thresholds that fell significantly from $-39.7 \pm 0.7$ in control to $-44.7 \pm 1.1$ in sensitized neurons (Table 1).

Contrary to the observation made after a long-term inflammation, this group of neurons showed a larger number of action potentials than controls to intracellular current pulses indicating an increased excitability (Fig. $4 B$ ). 
A
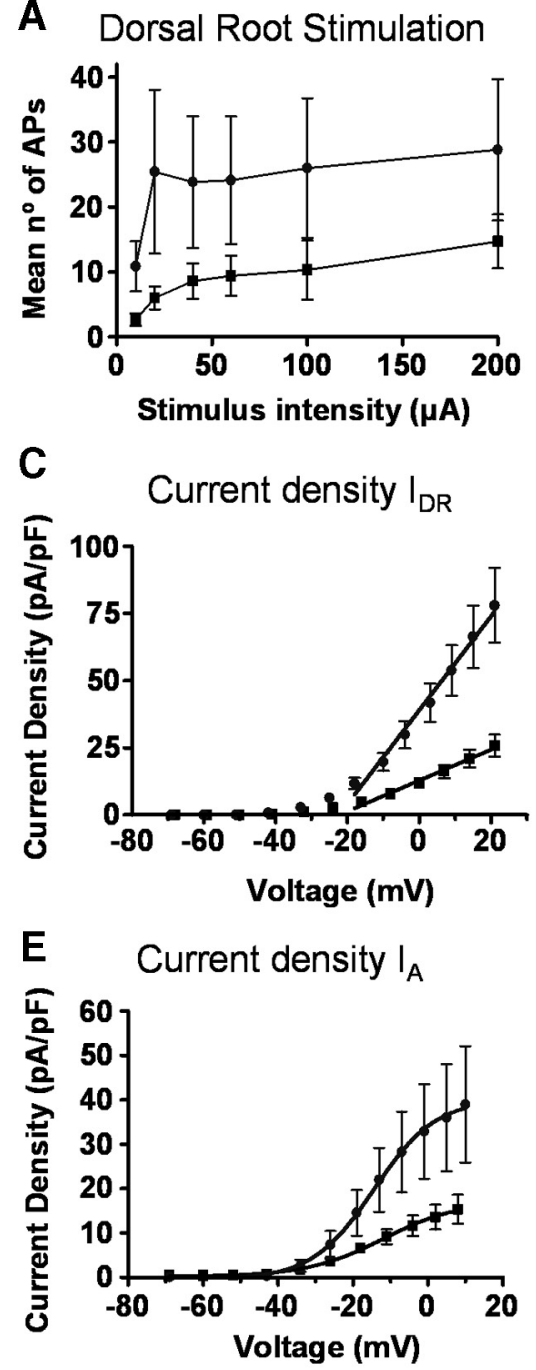

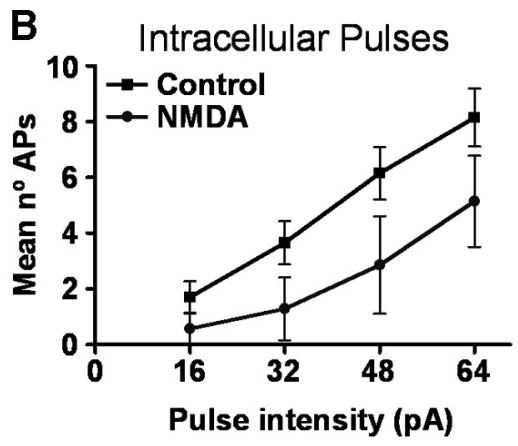

D

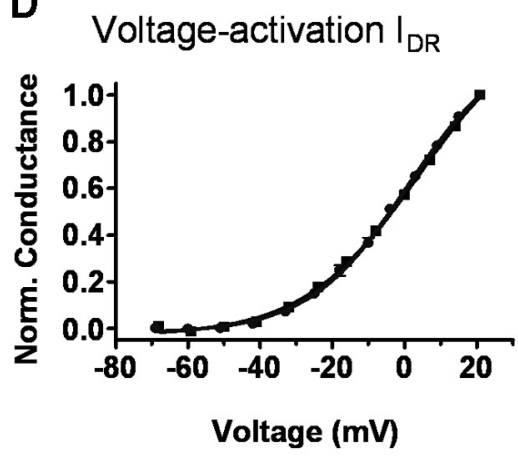

F Activation//nactivation $I_{A}$

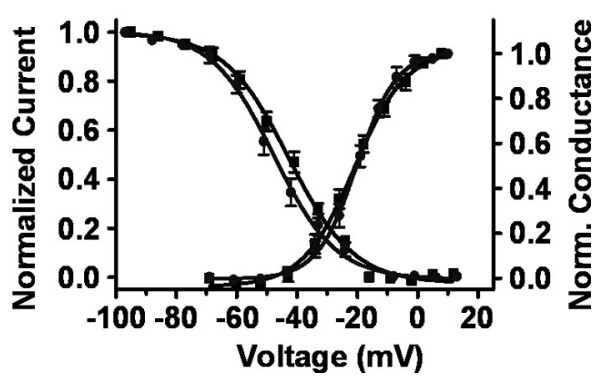

Figure 3. Effects of NMDA superfusion. Graphs in $\boldsymbol{A}$ and $\boldsymbol{B}$ show pooled data on action potentials elicited by dorsal root stimulation and intracellular current pulses in control (black symbols, $n=19$ ) and NMDA-treated neurons (gray symbols, $n=7$ ). Differences between groups were significant (ANOVA, $p<0.001$ and $p<0.01$, respectively) and resemble those obtained after a long-term inflammation (see Fig. 1). Current densities for sustained ( $C, n=21$ in control and $n=14$ after NMDA) and transient potassium currents $\left(\boldsymbol{E}, n=18\right.$ in control and $n=11$ after NMDA) increased significantly after NMDA treatment $\left(I_{\mathrm{DR}}\right.$, slope of straight line fit, $p<0.01 ; I_{A}$, Slope of Boltzmann fit, $\left.p<0.05\right)$. Graph in $\boldsymbol{D}$ shows no effect of NMDA treatment on the activation curve for $I_{D R}$ as obtained form the same sample of neurons included in $\boldsymbol{C}$. Neither the inactivation $(\boldsymbol{F}$, left axis) nor the activation curves ( $\boldsymbol{F}$, right axis) for transient currents were changed by NMDA treatment.

Voltage-clamp recordings performed under short-term inflammation conditions showed no changes in current density for transient and sustained potassium currents. However, the voltage activation curve for $I_{\mathrm{DR}}$ showed a significant shift to the left. In addition, the slope of the voltage-dependent inactivation curve for $I_{\mathrm{A}}$ was smaller than in control (Fig. 4, Table 2).

This last experiment indicates that the intrinsic excitability of dorsal horn neurons increases shortly after a peripheral inflammation and that the $I_{\mathrm{A}}$ may contribute toward this effect. In addition, it shows that changes in synaptic activity and in $I_{\mathrm{DR}}$ are developing at this early stage after inflammation.

\section{Discussion}

Our experiments support previous observations indicating that synaptic mechanism are fundamental to the development of central sensitization (Baba et al., 1999; Nakatsuka et al., 1999). In our mice model certain synaptic changes, like the decrease in synaptic latency, occur shortly after inflammation and are maintained in the longer term. In contrast, other factors that may depend upon synaptic strengthening, like spontaneous firing, needed longer developing times. However, the major contribution of the present experiments is to show changes of intrinsic excitability in dorsal horn neurons during central sensitization which may contribute to shape the final response of the system.

Shortly after inflammation, the overall neuronal excitability is increased as evidenced by the large number of action potentials fired to depolarizing current pulses. The ionic mechanisms underlying this short-term increase in excitability may be complex. The present experiments show that after short-term inflammation, the inactivation curve for transient potassium currents of the A-type has a lower slope than that observed under control conditions while complete inactivation occurs at similar voltages in both groups. This means that the larger difference between the inactivating curves affects the potentials at which current starts inactivating. For example, at resting potential $(\sim-60 \mathrm{mV}), \sim 80 \%$ of channels in control neurons are available for activation whereas shortly after inflammation only a $60 \%$ of channels are available. In practical terms this change equates to a relaxation of a brake on excitability at physiologically meaningful potentials.

This current is modulated via ERK (Hu et al., 2003, 2006) which, in turn, is activated immediately after inflammation for a brief period of time (Galan et al., 2002). However the exact nature of the primary signal that triggers this process is not known. NMDA treatment induces opposite changes in $I_{\mathrm{A}}$ suggesting that the increased excitability seen in the early stage of sensitization is not an activity-dependent change but may depend on a more specific signal. Changes in A-type currents have been shown to follow inflammation in dorsal root ganglion cells suggesting that this is a crucial current to alter the functioning of the nociceptive system (Yoshimura and de Groat, 1999; Stewart et al., 2003; Dang et al., 2004; Takeda et al., 2006; Xu et al., 2006; Wang et al., 2007).

In addition to $I_{\mathrm{A}}$, it is likely that other currents may contribute toward increasing membrane excitability at this early stage in the development of central sensitization. Our experiments show that firing threshold falls, and this suggests the involvement of sodium currents as well. In addition, the overall increase in excitability may occur while stabilizing changes are emerging. In our shortterm inflammation experiments the activation curve for the $I_{\mathrm{DR}}$ was steeper than normal allowing this current to enhance its functionality. 
At longer times following inflammation, we report a decreased excitability in dorsal horn neurons evidenced by a decrease in the number of action potentials fired to depolarizing current pulses. This depressed excitability is likely caused, at least in part, by the observed changes in sustained outward potassium currents. After carrageenan injection, current density was significantly increased, and the activation curve showed a rightward shift at mildly depolarized membrane potentials. While an increase in current density may result in a direct reduction of excitability, the observed shift in the activation curve may allow this current to act more selectively upon large depolarizations reducing repetitive firing (Misonou et al., 2005).

A reduction of delayed rectifier type currents has been reported in dorsal root ganglion neurons as a consequence of inflammatory stimuli which may contribute to increase the excitability of primary afferents (Stewart et al., 2003). The increase of this current in spinal cord neurons is more difficult to understand in functional terms.

Experimental evidence emerging in later years suggests that neuronal excitability may be modulated in a homeostatic way to compensate for changes in the level of synaptic activity (Davis and Bezprozvanny, 2001; Turrigiano and Nelson, 2004). Sustained excitation produced by exogenous glutamate enhances A-type currents decreasing neuronal excitability in cortical neurons (Shen et al., 2008, but see Lei et al., 2008 in hippocampal neurons), and shifts the voltage activation curve of sustained currents toward more hyperpolarized potentials in hippocampal neurons ( $\mathrm{Mi}$ sonou et al., 2004).

NMDA receptors are important mediators of spinal nociceptive transmission (King and Lopez-Garcia, 1993) implicated in the generation of windup, LTP, and other forms pain plasticity (Liu and Sandkühler, 1995; Herrero et al., 2000; Woolf and Salter, 2000), and their activation can lead to modulation of potassium currents (Lei et al., 2008; Mulholland et al., 2008; Shen et al., 2008). In our experiments, incubation of the spinal cord with NMDA produced an increase in the gain of the dorsal horn together with a reduced excitability of the neurons in the area, a state remarkably similar to that generated by a long-term inflammation. In addition, NMDA treatment increased the density of sustained and transient potassium currents. These results suggest that homeostatic modulation of potassium currents may occur in the spinal cord in situations of sustained excitatory activity.

Our experiments show that the $I_{\mathrm{DR}}$ is increased after a longterm inflammation and that this change may start shortly after

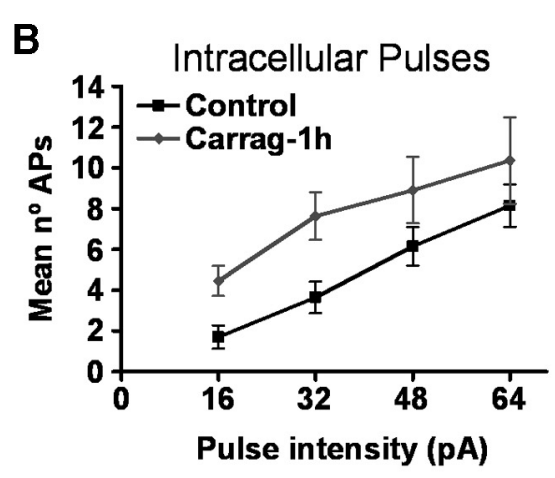

D

\section{Voltage-activation $I_{D R}$}

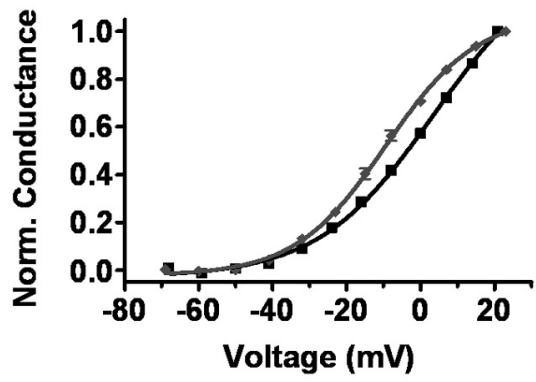

F Activation/Inactivation $\mathrm{I}_{\mathrm{A}}$

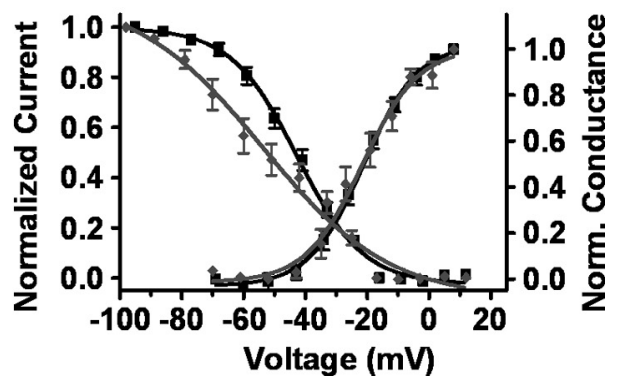

Figure 4. Effects of a short-term inflammation (1 h). Graphs in $\boldsymbol{A}$ and $\boldsymbol{B}$ show pooled data on action potentials elicited by dorsal root stimulation and intracellular current pulses in control (black symbols, $n=19$ ) and after a short-term inflammation (gray symbols, $n=11$ ). Responses to dorsal root stimulation were increased (ANOVA, $p<0.001$ ) compared with controls as for a longer-term treatment. Responses to intracellular current pulses were also increased (ANOVA, $p<0.001$ ) in contrast to results obtained for a longer-term inflammation (compare with Fig. 1). C, Compared to control ( $n=21)$, sustained currents did not show oltage activation curve was significantly displaced to the left $\left(\boldsymbol{D}, V_{50}\right.$ and slope of Boltzmann fit, $\left.p<0.001\right)$. $\boldsymbol{E}, \boldsymbol{F}$, Current density and voltage-dependent activation $(\boldsymbol{F}$, right axis) curves for transient currents $(n=7)$ were not significantly different from controls $(n=18)$. However, the voltage-dependent inactivation curve for transient currents ( $\boldsymbol{F}$, left axis) had a smaller slope in treated than in control groups (slope of Boltzmann fit, $p<0.05$ ).

inflammation following the development of synaptic changes. In the absence of further proof, we propose as a plausible working hypothesis that the decreased excitability and the increased density of sustained potassium currents observed after a long-term inflammation constitute a homeostatic change in response to an excess synaptic activity.

In conclusion, the present work shows biphasic changes in intrinsic excitability associated to central sensitization of dorsal horn neurons. In an early phase, specific signals may modulate ionic conductances like the $I_{\mathrm{A}}$ to increase neuronal excitability allowing for a rapid response of the system. In the long run however, a depression of neuronal excitability prevails by modifying conductances such as the $I_{\mathrm{DR}}$ in what may constitute a homeostatic change triggered by sustained excitatory activity produced by inflammation. 


\section{References}

Baba H, Doubell TP, Woolf CJ (1999) Peripheral inflammation facilitates abeta fiber-mediated synaptic input to the substantia gelatinosa of the adult rat spinal cord. J Neurosci 19:859-867.

Dang K, Bielefeldt K, Gebhart GF (2004) Gastric ulcers reduce A-type potassium currents in rat gastric sensory ganglion neurons. Am J Physiol Gastrointest Liver Physiol 286:G573-G579.

Davis GW, Bezprozvanny I (2001) Maintaining the stability of neural function: a homeostatic hypothesis. Annu Rev Physiol 63:847-869.

Desai NS, Rutherford LC, Turrigiano GG (1999) Plasticity in the intrinsic excitability of cortical pyramidal neurons. Nat Neurosci 2:515-520.

Galan A, Lopez-Garcia JA, Cervero F, Laird JM (2002) Activation of spinal extracellular signaling-regulated kinase- 1 and -2 by intraplantar carrageenan in rodents. Neurosci Lett 322:37-40.

Hedo G, Laird JM, Lopez-Garcia JA (1999) Time-course of spinal sensitization following carrageenan-induced inflammation in the young rat: a comparative electrophysiological and behavioural study in vitro and in vivo. Neuroscience 92:309-318.

Herrero JF, Laird JM, López-García JA (2000) Wind-up of spinal cord neurones and pain sensation: Much ado about something? Prog Neurobiol 61:169-203.

Hu HJ, Glauner KS, Gereau RW 4th (2003) ERK integrates PKA and PKC signaling in superficial dorsal horn neurons. I. Modulation of A-type K+ currents. J Neurophysiol 90:1671-1679.

Hu HJ, Carrasquillo Y, Karim F, Jung WE, Nerbonne JM, Schwarz TL, Gereau RW 4th (2006) The kv4.2 potassium channel subunit is required for pain plasticity. Neuron 50:89-100.

Jerng HH, Pfaffinger PJ, Covarrubias M (2004) Molecular physiology and modulation of somatodendritic A-type potassium channels. Mol Cell Neurosci 27:343-369.

Ji RR, Woolf CJ (2001) Neuronal plasticity and signal transduction in nociceptive neurons: implications for the initiation and maintenance of pathological pain. Neurobiol Dis 8:1-10.

King AE, Lopez-Garcia JA (1993) Excitatory amino acid receptor-mediated neurotransmission from cutaneous afferents in rat dorsal horn in vitro. J Physiol 472:443-457.

Lei Z, Deng P, Xu ZC (2008) Regulation of Kv4.2 channels by glutamate in cultured hippocampal neurons. J Neurochem 106:182-192.

Liu XG, Sandkühler J (1995) Long-term potentiation of C-fiber-evoked potentials in the rat spinal dorsal horn is prevented by spinal $N$-methyl-Daspartic acid receptor blockage. Neurosci Lett 191:43-46.

Lopez-Garcia JA, King AE (1994) Membrane properties of physiologically classified rat dorsal horn neurons in vitro: correlation with cutaneous sensory afferent input. Eur J Neurosci 6:998-1007.

Martinez-Gomez J, Lopez-Garcia JA (2005) Electrophysiological and pharmacological characterisation of ascending anterolateral axons in the in vitro mouse spinal cord. J Neurosci Methods 146:84-90.

Misonou H, Mohapatra DP, Park EW, Leung V, Zhen D, Misonou K, Anderson AE, Trimmer JS (2004) Regulation of ion channel localization and phosphorylation by neuronal activity. Nat Neurosci 7:711-718.

Misonou H, Mohapatra DP, Trimmer JS (2005) Kv2.1: a voltage-gated K+ channel critical to dynamic control of neuronal excitability. Neurotoxicology 26:743-752.

Mulholland PJ, Carpenter-Hyland EP, Hearing MC, Becker HC, Woodward JJ, Chandler LJ (2008) Glutamate transporters regulate extrasynaptic NMDA receptor modulation of Kv2.1 potassium channels. J Neurosci 28:8801-8809.

Nakatsuka T, Park JS, Kumamoto E, Tamaki T, Yoshimura M (1999) Plastic changes in sensory inputs to rat substantia gelatinosa neurons following peripheral inflammation. Pain 82:39-47.

Rivera-Arconada I, Lopez-Garcia JA (2005) Effects of M-current modulators on the excitability of immature rat spinal sensory and motor neurones. Eur J Neurosci 22:3091-3098.

Rudy B (1988) Diversity and ubiquity of K channels. Neuroscience 25:729-749.

Sandkühler J, Benrath J, Brechtel C, Ruscheweyh R, Heinke B (2000) Synaptic mechanisms of hyperalgesia. Prog Brain Res 129:81-100.

Shen B, Zhou K, Yang S, Xu T, Wang Y (2008) The Kv4.2 mediates excitatory activity-dependent regulation of neuronal excitability in rat cortical neurons. J Neurochem 105:773-783.

Stewart T, Beyak MJ, Vanner S (2003) Ileitis modulates potassium and sodium currents in guinea pig dorsal root ganglia sensory neurons. J Physiol 552:797-807.

Storm JF (1990) Potassium currents in hippocampal pyramidal cells. Prog Brain Res 83:161-187.

Takeda M, Tanimoto T, Ikeda M, Nasu M, Kadoi J, Yoshida S, Matsumoto S (2006) Enhanced excitability of rat trigeminal root ganglion neurons via decrease in A-type potassium currents following temporomandibular joint inflammation. Neuroscience 138:621-630.

Turrigiano GG, Nelson SB (2004) Homeostatic plasticity in the developing nervous system. Nat Rev Neurosci 5:97-107.

van Welie I, van Hooft JA, Wadman WJ (2006) Background activity regulates excitability of rat hippocampal CA1 pyramidal neurons by adaptation of a K+ conductance. J Neurophysiol 95:2007-2012.

Wang JG, Strong JA, Xie W, Zhang JM (2007) Local inflammation in rat dorsal root ganglion alters excitability and ion currents in small-diameter sensory neurons. Anesthesiology 107:322-332.

Woolf CJ (1983) Evidence for a central component of post-injury pain hypersensitivity. Nature 306:686-688.

Woolf CJ (2007) Central sensitization: uncovering the relation between pain and plasticity. Anesthesiology 106:864-867.

Woolf CJ, Costigan M (1999) Transcriptional and posttranslational plasticity and the generation of inflammatory pain. Proc Natl Acad Sci U S A 96:7723-7730.

Woolf CJ, Salter MW (2000) Neuronal plasticity: increasing the gain in pain. Science 288:1765-1769.

Xu GY, Winston JH, Shenoy M, Yin H, Pasricha PJ (2006) Enhanced excitability and suppression of A-type $\mathrm{K}+$ current of pancreas-specific afferent neurons in a rat model of chronic pancreatitis. Am J Physiol Gastrointest Liver Physiol 291:G424-G431.

Yoshimura N, de Groat WC (1999) Increased excitability of afferent neurons innervating rat urinary bladder after chronic bladder inflammation. J Neurosci 19:4644-4653. 